\title{
The role of qualitative elastography in thyroid nodule evaluation: exploring its target populations
}

\author{
Jakob W. Kist ${ }^{1} \cdot$ Sjoerd Nell $^{1}$ - Bart de Keizer ${ }^{2} \cdot$ Gerlof D. Valk $^{3}$ • \\ Inne H. M. Borel Rinkes ${ }^{1} \cdot$ Menno R. Vriens ${ }^{1}$
}

Received: 15 April 2015/Accepted: 23 May 2015/Published online: 10 June 2015

(C) Springer Science+Business Media New York 2015

Thyroid nodules are frequently found and pose a dilemma to the clinician, as only a few harbor a malignancy, and the majority of nodules are benign. The standard work-up of thyroid nodules consists of ultrasound and fine needle aspiration (FNA), both having their limitations $[1,2]$. In particular, ultrasound lacks criteria for determining whether a nodule is malignant [3]. FNA shows good sensitivities and specificities, but inconclusive and indeterminate results are frequently found, resulting in the need for a diagnostic thyroid lobectomy to obtain a final diagnosis [4]. In the long lasting search to reduce the number of invasive diagnostic procedures, real-time qualitative elastography has been proposed to fulfill this need. Elastography determines the elasticity of the thyroid nodule. Soft nodules are assumed to be benign, whereas hard nodules are considered to be malignant. Qualitative elastography represents the elasticity of the nodule in a colored image projected over the ultrasound image. Multiple elasticity scoring systems are used, which makes reviewing literature challenging. Most common is the 4-point scale developed by Asteria et al., in which elastography 1 (ES 1) is assigned to nodules with elasticity in the entire nodule, ES 2 is assigned to

Jakob W. Kist and Sjoerd Nell have contributed equally to the manuscript.

Menno R. Vriens

mvriens@umcutrecht.nl

1 Department of Surgical Oncology and Endocrine Surgery, University Medical Center Utrecht, Room G04.228, Heidelberglaan 100, 3584 CX Utrecht, The Netherlands

2 Department of Radiology and Nuclear Medicine, University Medical Center Utrecht, Utrecht, The Netherlands

3 Department of Endocrinology, University Medical Center Utrecht, Utrecht, The Netherlands nodules with elasticity in a large portion of the nodule, ES 3 is assigned to nodules with stiffness in a large portion of the nodule, and ES 4 is assigned to hard nodules. A cut-off between ES 2 and ES 3 is widely accepted to discriminate benign from malignant nodules [5].

Studies on thyroid nodule elastography have focused on different target populations: (1) patients referred for FNA with the aim to reduce the number of FNAs, and (2) patients with an indeterminate FNA result (i.e., Bethesda classification III or IV), with the aim to reduce the number of futile lobectomies.

Recently, we performed a meta-analysis of studies that investigated the first population: patients referred for FNA. The aim of this study was to determine whether elastography could determine the nature of thyroid nodules and thereby identify those that require further analysis by FNA [6]. In this study, analyzing twenty reports including 3908 nodules, two different cut-offs were examined. The first was the standard cut-off between ES 2 and 3. The second used a cut-off between ES 1 and 2, meaning that only the completely soft nodules were considered benign and the rest of the nodules as potentially malignant. Both cut-offs showed that elastography is an excellent tool to diagnose benignity, with a respective negative predictive value (NPV) of 97 and $99 \%$. Based on these outcomes, it was concluded that in these cases FNA could be omitted safely. However, considering the modest positive predictive value (PPV) of only $40 \%$ of elastography, this implies that any nodule with an elastography score above ES 2 requires further analysis [6].

In the current issue of Endocrine, Trimboli et al. published an extensive review and meta-analysis on nodules with an indeterminate FNA [7]. Although the majority of these nodules are benign, around one in four harbors a malignancy [4]. Diagnostic lobectomies are often 
performed to obtain conclusive histology. To reduce the number of futile surgeries, it would be of great interest to find a tool that can determine which nodules should be operated on. In this meta-analysis, almost 500 thyroid nodules with indeterminate FNA derived from eight studies were analyzed. Three of these studies especially focused on these particular nodules, while the rest consisted of a subgroup of studies investigating the overall performance of elastography. All studies were relatively small with a maximum of 142 nodules included. The pooled estimated diagnostic parameters are disappointing with a sensitivity of $69 \%$, a specificity of $75 \%$, and a respective NPV and PPV of 82 and $63 \%$. The heterogeneity between the studies was moderate to high in all diagnostic parameters $\left(I^{2} 68-95 \%\right)$. Taken together, these findings lead to the justified conclusion that, for this population, qualitative elastography is not able to guide the clinician in his decision-making and that a diagnostic lobectomy should be performed to obtain a final diagnosis in all cases.

There is an apparent difference between the outcome of our meta-analysis and the meta-analysis of Trimboli et al. $[6,7]$. The excellent NPV of $97 \%$ for elastography in nodules referred for FNA was not reproduced in nodules with an indeterminate FNA. Trimboli et al. found a NPV of $82 \%$.

The main difference between both meta-analyses is obviously the target population: nodules referred for FNA versus nodules with an indeterminate FNA outcome. However, it is unclear why this would influence the NPV. The higher a priori chance of malignancy in the metaanalysis of Trimboli et al. might have lowered the NPV slightly, but this does not explain this significant difference. Other potential causes are the relatively small number of included studies, the nodules analyzed per study, and the overall number of analyzed nodules. Moreover, the different outcomes could be based on the high heterogeneity of the included studies in the meta-analysis by Trimboli. This might be caused by publication bias, as suggested by the authors, but as well by the classification of nodules as indeterminate, since several studies showed that FNA cytopathology is complicated and requires expertise [8]. The included indeterminate nodules could be influenced by this phenomenon and, therefore, not be as homogeneous as desirable. Based on the currently published data, a meta-analysis of the studies included by Trimboli et al. with an alternative cut-off (between ES 1 and 2), like performed in our meta-analysis, was not possible. In the future, studies focusing on this alternative cutoff should be performed.

At this point, it is uncertain whether the described modest diagnostic performance of qualitative elastography of thyroid nodules with an indeterminate FNA represents the ground truth or that future studies specifically focusing on the indeterminate thyroid nodules will reveal better results. We propose that such future studies should be done using the novel, more advanced quantitative elastography techniques, such as shear wave elastography and acoustic radiation force impulse imaging. These techniques have been shown to have promising accuracy results for malignancy, said to be less operator dependent and more consistent, although they have not been studied extensively in indeterminate nodules specifically [9]. Another topic of future studies could aim to evaluate the combination of ultrasound and elastography scoring systems to guide the necessity of FNA in the ES positive nodules $[10,11]$.

In conclusion, qualitative elastography can replace FNAs in patients with (nearly) complete soft thyroid nodules, but its role in guiding clinicians in case of indeterminate cytology remains to be determined.

Conflict of interest The authors declare that there are no conflicts of interest.

\section{References}

1. Y.Y. Tee, A.J. Lowe, C.A. Brand, R.T. Judson, Fine-needle aspiration may miss a third of all malignancy in palpable thyroid nodules: a comprehensive literature review. Ann. Surg. 246, 714-720 (2007)

2. T.D. Samulski, C. Shutty, V.A. LiVolsi, K. Montone, Z. Baloch, The reliability of thyroid nodule ultrasound features and size to predict malignancy in fine needle aspiration specimens: practical utility for the evaluating pathologist. Diagn. Cytopathol. 43, 471-477 (2015)

3. J.P. Brito, M.R. Gionfriddo, A. Al Nofal, K.R. Boehmer, A.L. Leppin, C. Reading, M. Callstrom, T.A. Elraiyah, L.J. Prokop, M.N. Stan, M.H. Murad, J.C. Morris, V.M. Montori, The accuracy of thyroid nodule ultrasound to predict thyroid cancer: systematic review and meta-analysis. J. Clin. Endocrinol. Metab. 99, 1253-1263 (2014)

4. M. Bongiovanni, A. Spitale, W.C. Faquin, L. Mazzucchelli, Z.W. Baloch, The Bethesda system for reporting thyroid cytopathology: a meta-analysis. Acta Cytol. 56, 333-339 (2012)

5. C. Asteria, A. Giovanardi, A. Pizzocaro, L. Cozzaglio, A. Morabito, F. Somalvico, A. Zoppo, US-elastography in the differential diagnosis of benign and malignant thyroid nodules. Thyroid 18, 523-531 (2008)

6. S. Nell, J.W. Kist, T.P.A. Debray, B. de Keizer, T.J. van Oostenbrugge, I.H.M.B. Rinkes, G.D. Valk, M.R. Vriens, Qualitative elastography can replace thyroid nodule fine-needle aspiration in patients with soft thyroid nodules. A systematic review and meta-analysis. Eur. J. Radiol. 84, 652-661 (2015)

7. P. Trimboli, G. Treglia, L. Guidobaldi, E. Saggiorato, G. Nigri, A. Crescenzi, F. Romanelli, F. Orlandi, S. Valabrega, R. Sadeghi, L. Giovanella, Clinical characteristics as predictors of malignancy in patients with indeterminate thyroid cytology: a meta-analysis. Endocrine 46, 52-59 (2014). doi:10.1007/s12020-013-0057-1

8. T. Davidov, S.Z. Trooskin, B.A. Shanker, D. Yip, O. Eng, Routine second-opinion cytopathology review of thyroid fine needle aspiration biopsies reduces diagnostic thyroidectomy. Surgery 148, 1294-1301 (2010) 
9. V. Cantisani, P. Lodise, H. Grazhdani, E. Mancuso, E. Maggini, G. Di Rocco, F. D’Ambrosio, F. Calliada, A. Redler, P. Ricci, C. Catalano, Ultrasound elastography in the evaluation of thyroid pathology. Current status. Eur J Radiol. 83, 420-428 (2014)

10. G. Russ, B. Royer, C. Bigorgne, A. Rouxel, M. Bienvenu-Perrard, L. Leenhardt, Prospective evaluation of thyroid imaging reporting and data system on 4550 nodules with and without elastography. Eur. J. Endocrinol. 168, 649-655 (2013)
11. P. Trimboli, R. Guglielmi, S. Monti, I. Misischi, F. Graziano, N. Nasrollah, S. Amendola, S.N. Morgante, M.G. Deiana, S. Valabrega, V. Toscano, E. Papini, Ultrasound sensitivity for thyroid malignancy is increased by real-time elastography: a prospective multicenter study. J. Clin. Endocrinol. Metab. 97, 4524-4530 (2012) 